\title{
KIF1A-related disorders in children: a wide spectrum of central and peripheral nervous system involvement
}

Authors: Tarishi Nemani*, ${ }^{1}$ Dora Steel ${ }^{*},{ }^{1,2}$ Marios Kaliakatsos, ${ }^{1}$ Catherine DeVile, ${ }^{1}$ Athina Ververi, ${ }^{3}$ Richard Scott, ${ }^{3}$ Spas Getov, ${ }^{5}$ Sniya Sudhakar, ${ }^{6}$ Alison Male, ${ }^{3}$ Kshitij Mankad, ${ }^{6}$ Genomics England Research Consortium, ${ }^{7}$ Francesco Muntoni, ${ }^{1,2}$ Mary M Reilly, ${ }^{4}$ Manju A Kurian, ${ }^{1,2}$ Lucinda Carr, ${ }^{1}$ Pinki Munot ${ }^{1}$

* Joint first authors

1. Department of Paediatric Neurology, Great Ormond Street Hospital, London, UK

2. UCL Great Ormond Street Institute of Child, London, UK

3. Department of Clinical Genetics, Great Ormond Street Hospital, London, UK

4. UCL Queen Square Institute of Neurology, London, UK

5. Department of Neurophysiology, Great Ormond Street Hospital, London, UK

6. Department of Radiology, Great Ormond Street Hospital, London, UK

7. Genomics England, Queen Mary University of London, UK

Running Title: Spectrum of KIF1A disorders in children

Word Count: 2145 


\begin{abstract}
Background and Aims

KIF1A-related disorders (KRD) were first described in 2011 and the phenotypic spectrum has subsequently expanded to encompass a range of central and peripheral nervous system involvement. Here we present a case series demonstrating the range of clinical, neurophysiological and radiological features which may occur in childhood-onset KRD.

\section{Methods}

We report on all the children and young people seen at a single large tertiary centre. Data was collected through a retrospective case-notes review.

\title{
Results
}

12 individuals from 10 families were identified. Eight different mutations were present, including four novel mutations. Two patients displayed a very severe phenotype including congenital contractures, severe spasticity and/or dystonia, dysautonomia, severe sensorimotor polyneuropathy and optic atrophy, significant white matter changes on brain MRI, respiratory insufficiency, and complete lack of neurodevelopmental progress. The remaining 10 patients represented a spectrum of severity with common features including a movement disorder with spasticity and/or dystonia, subtle features of dysautonomia, sensory axonal neuropathy, varying degrees of optic atrophy and of learning and/or behavioural difficulties, and subtle or absent - but sometimes progressive - changes in white matter on MRI. Epilepsy was common among the more severely-affected children.

\section{Interpretation}

This case series demonstrates that KRD comprise a range of neurological disorders, with both the milder and the more severe forms combining central and peripheral (including autonomic) nervous system deficits. 


\section{Keywords}

KIF1A; hereditary spastic paraparesis; neuropathy; dysautonomia, dystonia

\section{Introduction}

The KIF1A gene encodes a protein in the kinesin family, involved in the anterograde axonal transport of membranous organelles, including synaptic vesicles. ${ }^{1}$ Pathogenic variants in KIF1A have been linked to a wide spectrum of neurological disorders. Initially, biallelic variants were identified in cases of hereditary spastic paraparesis (HSP) ${ }^{2,3}$ and hereditary sensory and autonomic neuropathy. ${ }^{4}$ Subsequently it became clear that heterozygous de novo variants could be associated with a severe neurodegenerative condition, sometimes overlapping with progressive encephalopathy with edema, hypsarrhythmia and optic atrophy (PEHO) syndrome..$^{5-8}$ Thirdly, heterozygous inherited variants associated with both pure and complicated HSP syndromes were reported. ${ }^{9-12}$

Despite this wide phenotypic spectrum, KIF1A-related disorders (KRD) as a whole remain extremely rare, and few neurologists will encounter many affected individuals in their careers. We report on a series of patients seen in a large tertiary centre, offering a description of the clinical, genetic, neurophysiological and radiological findings seen in this condition.

\section{Methods}

The setting was a paediatric neurology department providing a tertiary/quaternary service. Patients with confirmed KRD were identified through a survey of Paediatric Neurology consultants caring for patients with motor difficulties. This covers the majority of the patients seen at our institution since the initial description of KRD in $2011 .^{2}$ 
Clinical information was collected by retrospective review of electronic clinical records, including clinical correspondence; genetic and other laboratory results; brain magnetic resonance imaging (MRI) scans and neurophysiology results.

Approximate Gross Motor Function Classification System (GMFCS) equivalent scores were assigned, where possible, based on a review of medical files.

Genetic testing was carried out at a number of centres including through the Deciphering Developmental Disorders study ${ }^{13}$ and the 100,000 Genomes Project. ${ }^{14}$ All mutation details refer to transcript NM_004321.6.

This study was approved by the Great Ormond Street Hospital for Children Research and Development Board with reference number 19NM23.

\section{Results}

12 individuals in 10 families with confirmed pathogenic KIF1A mutations were identified. 5/12 were male and the mean age at the last recorded clinical review was 11.6 years (range 6 months to 21 years). Their clinical, radiological and neurophysiological characteristics are summarised in Table 1. One individual (8B) was identified through diagnosis in her sibling, having never herself been a patient at our institution.

\section{Genetics}

All patients in our cohort had single heterozygous variants, with no biallelic mutations identified. In one family autosomal dominant inheritance was confirmed, with the mother experiencing symptoms similar to the two affected children. In all other families de novo 
inheritance was either confirmed ( 6 cases) or considered highly likely in view of the severity of the phenotype.

Eight variants were identified across the 10 families, with two unrelated patients sharing the c.757G $>\mathrm{A}$ variant, which is known to be associated with severe early-onset disease ${ }^{15}$ and two families sharing c.206C $>$ T. Four of the eight variants were either previously reported or known to ClinVar ${ }^{16}$ and four were novel: c.32G>A, p.Arg11GIn; c.556G>T, p.Val186Phe; c.967T>C, p.Ser323Pro; and c.1038-1G>A, p.?. The latter is the first pathogenic splicing variant reported in KRD.

\section{Clinical Data}

Two unrelated children (Patients 1 and 2) presented at or before birth with reduced foetal movements and multiple limb contractures suggestive of arthrogryposis. They also had additional congenital anomalies: Patient 1 had rocker-bottom feet and Patient 2 had a pelvic kidney. Both shared the severe c.757G >A variant. They had marked limb spasticity and dystonia, combined with a severe polyneuropathy, marked dysautonomic symptoms and respiratory insufficiency. One other child (Patient 6) who also presented antenatally with reduced foetal movements, however, went on to have a milder course.

All the remaining children (except $8 \mathrm{~B}$, where details of initial presentation are unknown) presented in the first two years of life with the primary presenting complaint being delayed psychomotor development.

The two profoundly-affected children made virtually no developmental progress, although Patient 2 did temporarily gain partial head control. Both died in infancy from respiratory illnesses, one aged six months and the other 23 months. 
Details of development are known for 9 of the remaining 10 children. Of these, 5/9 had moderate to severe learning difficulties (LD), 2/9 had mild LD, and 2/9 had normal cognition. A broad correlation was observed between severity of physical disability and severity of LD. $4 / 9$ had a diagnosis of autism spectrum disorder (ASD); $2 / 9$ had attention deficit hyperactivity disorder (ADHD); and 4/9 displayed challenging and/or obsessional behaviour. Every child had some degree of learning and/or neuropsychiatric difficulty. Cognitive regression, however, was not reported in any of our patients.

All patients had some degree of impairment in their motor skills at their latest review. Leaving aside the two children who died in infancy, this ranged from ability to perform standing transfers with assistance only (estimated GMFCS IV-V) to walking and running despite some falls and reduced stamina (estimated GMFCS II). No patient experienced a period of completely normal development.

Eight patients had more than one recorded assessment of their motor abilities, and in all but one of these (patient 4) deterioration over time was seen.

On examination, limb spasticity was detected in $8 / 12$ patients and $6 / 8$ of these also had dystonia, varying from severe generalised dystonia in patients 1 and 2 to subtle upper limb posturing in patient $8 A$. Surprisingly, in $4 / 12$ patients neither spasticity nor dystonia was detected, although two of these were noted to have brisk deep tendon reflexes and one also had upgoing plantar responses. Two patients also had some signs of impaired cerebellar function.

The two profoundly-affected children had features of severe dysautonomia, including urinary retention, temperature instability and in the case of Patient 1 gastrointestinal dysfunction sufficiently severe to require parenteral nutrition. 
Among the other patients, more subtle dysautonomic symptoms were observed, including incontinence of urine in $3 / 10$ (accompanied by incontinence of stool in one, and constipation in the other two) and poor perfusion of the feet in 3/10.

One patient (8B) had epilepsy which could be attributable to causes other than her KRD, as she had had bacterial meningitis in early childhood. $8 / 11(73 \%)$ of the remaining patients had epilepsy, and this was highly variable in both nature and onset. Notably, although both $5 \mathrm{~A}$ and $5 \mathrm{~B}$, who were monozygotic twins, developed epilepsy, the onset in $5 \mathrm{~B}$ was in early childhood, while in $5 \mathrm{~A}$ it was in the late teens.

Both the profoundly-affected infants had onset of epilepsy in the first year of life (infantile spasms in patient 1 , and seizures associated with apnoea and eye deviation in patient 2). Seizure types in the other patients included generalised tonic-clonic seizures, behavioural arrest, myoclonic jerks and focal motor seizures with impaired awareness. In all patients it was possible to achieve acceptable seizure control using anti-epileptic medication. As with LD, there appeared to be an association between presence of epilepsy and severity of motor impairment.

Some degree of optic atrophy (OA) was present in 7/12 patients. Children with severe early-onset motor impairment and severe LD were more likely also to have OA. The two profoundly-affected infants had severe OA and did not develop any visual behaviour. Two other children also had a significant post-retinal visual impairment. Nystagmus was noted in one child. Of the five patients with the least severe motor impairments, however, none had any recorded optic atrophy or other significant visual pathology.

11/12 patients were investigated with peripheral nerve electrodiagnostic studies, and these were abnormal in $8 / 11$ cases $(73 \%)$. The degree of abnormality varied from profound axonal neuropathy with absent sensory and severely attenuated motor responses to a more 
moderate sensorimotor axonal neuropathy. There were five cases of predominantly sensory neuropathy and none in which motor neuropathy was more prominent. No child, however, presented with symptoms of sensory neuropathy and in no case was a sensory deficit noted in the initial examination of any patient, although in one family (5) the parents reported apparent insensitivity to pain.

Brain MRI was available for $11 / 12$ individuals and $8 / 11$ of these showed relevant abnormalities (see Figure 1). There was a clear correlation between clinical severity and degree of radiological abnormality, with the most severely-affected children demonstrating features including callosal dysgenesis, cerebellar hypoplasia, optic atrophy and white matter signal changes. In less severely-affected children, white matter changes were milder or absent. The monozygotic twins (5A, 5B) also showed bulky brainstems with symmetrical paramedian bumps. The most consistent radiological feature was cerebellar volume loss: this was found in all cases where the MRI was abnormal.

\section{Discussion}

The previously reported phenotypes of KRD include pure HSP, ${ }^{2,9}$ HSP complicated by cerebellar atrophy, intellectual disability and/or axonal neuropathy, ${ }^{11,17,18}$ and also severe and often fatal neonatal presentations..$^{5,8}$ (The pure recessive HSAN phenotype appears to have been described only once. ${ }^{4}$ )

Our cohort includes two children with the severe neonatal phenotype. This syndrome has been characterised in various ways in previous reports, including as progressive neurodegeneration, ${ }^{6}$ progressive encephalopathy with brain atrophy, ${ }^{8}$ and PEHO syndrome. ${ }^{5,15}$ The features - both in previous reports and in our cases - include spasticity, severe optic atrophy, epilepsy (not necessarily limited to infantile spasms), developmental 
stasis, profound dysautonomia and ultimately death. Although "mixed tone" has been mentioned only in one previously published patient, ${ }^{7}$ infants with the severe phenotype in our cohort had very marked dystonia as well as spasticity. As dystonia was also found in three of our less severely affected young people, this probably represents and under-recognised clinical features of KRD across the spectrum.

All the other patients in our cohort fall into the broad category of "complicated HSP". The absence of pure HSP may reflect case ascertainment bias, as it is more likely to present in adulthood. ${ }^{12}$ They share many common features with previously reported cohorts, ${ }^{7,12}$ including slowly-progressive upper motor neuron deficits (together with the less-described extrapyramidal features), accompanied by a sensory and autonomic axonal neuropathy, a variable degree of developmental or neuropsychiatric impairment, and often also epilepsy and/or optic atrophy. Our cohort experienced a higher prevalence of psychiatric and/or behavioural difficulties than has previously been reported, including challenging behaviour in three patients and ADHD in another two.

All the genetic variants in our cohort - and the large majority of those previously published with any KIF1A phenotype - occur within the kinesin motor domain. While a small number of pathogenic variants have been reported outside this region, these are generally protein-truncating rather than missense variants, and may therefore be expected to have a more disruptive effect on the protein as a whole..$^{4,12,17}$ Variants associated with the most severe phenotype appear to cluster around certain amino acids, ${ }^{8}$ although the reason for this genotype-phenotype correlation remains unclear. The severe neonatal phenotype appears always to occur de novo (as expected in lethal autosomal dominant conditions without significant variable penetrance) while milder dominant phenotypes may occur either de novo or with familial inheritance. 
Other paediatric presentations of KRD may well exist: for example, a Rett-like phenotype has recently been described. ${ }^{19} \mathrm{KRD}$ is therefore probably still under-recognised and under-diagnosed, particularly as the gene is not included on some widely-used diagnostic genetic panels for neuropathy, dystonia ${ }^{20}$ or for arthrogryposis, ${ }^{21}$ even though these can be important presenting symptoms. Although characteristic MRI or electrophysiological findings are present in some cases, the diagnosis of KRD cannot be excluded on the basis of these tests, as they may be normal - vide the near-normal neonatal MRI of our Case 2, who was already severely symptomatic at the time.

\section{Conclusion}

Most patients presenting with KRD in childhood will either experience a severe neonatal-onset phenotype or fall somewhere within the spectrum of complicated HSP. The finding of a sensory-predominant axonal neuropathy in combination with spasticity and/or dystonia and any degree of developmental delay should prompt consideration of KRD, especially if autonomic features are also present.

Dystonia has not previously been well-recognised as a feature of KRD but was significant in our neonatal-onset cases, and also present in some older children. As dystonia is not found in most forms of HSP or inherited neuropathies, this may assist in diagnosis.

Clinicians should be aware, however, that genetic panels targeted specifically at one of the presenting features of this polymorphous condition, such as dystonia or arthrogryposis, may currently miss the diagnosis. Although characteristic MRI findings will develop in many cases, they are not present in all individuals, and may be absent or equivocal at birth even in 
the most severely-affected children. Under-diagnosis or delayed diagnosis of KRD therefore remains a real risk.

The neonatal form of KRD is clearly devastating, but even the "milder" paediatriconset forms are complex multisystem disorders which can have far-reaching effects on patients' lives, including progressive deterioration in mobility (resulting from a combination

of upper motor neuron deficits, neuropathy and extrapyramidal symptoms), visual impairment, bladder and bowel dysfunction, neurodevelopmental impairment, and epilepsy.

Much more remains to be learned about the natural history and progression of KRD at all degrees of severity, and greater understanding of the genotype-phenotype correlation would allow for more accurate counselling to be given to families. Meanwhile, clinicians treating patients with KRD should be aware of the complexity of the disorder, and the potential for combined involvement of the central, peripheral and autonomic nervous systems.

\section{Acknowledgements}

DS and MAK are funded by an NIHR Research Professorship. This study makes use of data generated by the DECIPHER community. A full list of centres who contributed to the generation of the data is available from http://decipher.sanger.ac.uk and via email from decipher@sanger.ac.uk. Funding for the project was provided by Wellcome.

This research was made possible through access to the data and findings generated by the 100,000 Genomes Project. The 100,000 Genomes Project is managed by Genomics England Limited (a wholly owned company of the Department of Health and Social Care). The 100,000 Genomes Project is funded by the National Institute for Health Research and NHS 
England. The Wellcome Trust, Cancer Research UK and the Medical Research Council have also funded research infrastructure. The 100,000 Genomes Project uses data provided by patients and collected by the National Health Service as part of their care and support.

\section{References}

1. Okada Y, Yamazaki H, Sekine-Aizawa Y, Hirokawa N. The neuron-specific kinesin superfamily protein KIF1A is a unique monomeric motor for anterograde axonal transport of synaptic vesicle precursors. Cell, 1995. 81(5):769-780.

2. Erlich Y, Edvardson S, Hodges E, Zenvirt S, Thekkat P, Shaag A, Dor T, Hannon GJ, Elpeleg O. Exome sequencing and disease-network analysis of a single family implicate a mutation in KIF1A in hereditary spastic paraparesis. Genome Res, 2011. 21(5):658-64.

3. Klebe S, Lossos A, Azzedine H, Mundwiller E, Sheffer R, Gaussen M, Marelli C, Nawara M, Carpentier W, Meyer V, Rastetter A, Martin E, Bouteiller D, Orlando L, Gyapay G, El-Hachimi KH, Zimmerman B, Gamliel M, Misk A, Lerer I, Brice A, Durr A, Stevanin G. KIF1A missense mutations in SPG30, an autosomal recessive spastic paraplegia: distinct phenotypes according to the nature of the mutations. Eur J Hum Genet, 2012. 20(6):645-9.

4. Rivière JB, Ramalingam S, Lavastre V, Shekarabi M, Holbert S, Lafontaine J, Srour M, Merner N, Rochefort D, Hince P, Gaudet R, Mes-Masson AM, Baets J, Houlden H, Brais B, Nicholson GA, Van Esch H, Nafissi S, De Jonghe P, Reilly MM, Timmerman V, Dion PA, Rouleau GA. KIF1A, an axonal transporter of synaptic vesicles, is mutated in hereditary sensory and autonomic neuropathy type 2. Am J Hum Genet, 2011. 89(2):219-30. 
5. Langlois S, Tarailo-Graovac M, Sayson B, Drögemöller B, Swenerton A, Ross CJ, Wasserman WW, van Karnebeek CD. De novo dominant variants affecting the motor domain of KIF1A are a cause of PEHO syndrome. Eur J Hum Genet, 2016. 24(6):949-53.

6. Okamoto N, Miya F, Tsunoda T, Yanagihara K, Kato M, Saitoh S, Yamasaki M, Kanemura Y, Kosaki K. KIF1A mutation in a patient with progressive neurodegeneration. J Hum Genet, 2014. 59(11):639-41.

7. Lee JR, Srour M, Kim D, Hamdan FF, Lim SH, Brunel-Guitton C, Décarie JC, Rossignol E, Mitchell GA, Schreiber A, Moran R, Van Haren K, Richardson R, Nicolai J, Oberndorff KM, Wagner JD, Boycott KM, Rahikkala E, Junna N, Tyynismaa H, Cuppen I, Verbeek NE, Stumpel CT, Willemsen MA, de Munnik SA, Rouleau GA, Kim E, Kamsteeg EJ, Kleefstra T, Michaud JL. De novo mutations in the motor domain of KIF1A cause cognitive impairment, spastic paraparesis, axonal neuropathy, and cerebellar atrophy. Hum Mutat, 2015. 36(1):69-78.

8. Esmaeeli Nieh S, Madou MR, Sirajuddin M, Fregeau B, McKnight D, Lexa K, Strober J, Spaeth C, Hallinan BE, Smaoui N, Pappas JG, Burrow TA, McDonald MT, Latibashvili M, LeshinskySilver E, Lev D, Blumkin L, Vale RD, Barkovich AJ, Sherr EH. De novo mutations in KIF1A cause progressive encephalopathy and brain atrophy. Ann Clin Transl Neurol, 2015. 2(6):623-35.

9. Ylikallio E, Kim D, Isohanni P, Auranen M, Kim E, Lönnqvist T, Tyynismaa H. Dominant transmission of de novo KIF1A motor domain variant underlying pure spastic paraplegia. Eur J Hum Genet, 2015. 23(10):1427-30.

10. Citterio A, Arnoldi A, Panzeri E, Merlini L, D'Angelo MG, Musumeci O, Toscano A, Bondi A, Martinuzzi A, Bresolin N, Bassi MT. Variants in KIF1A gene in dominant and sporadic forms of hereditary spastic paraparesis. J Neurol, 2015. 262(12):2684-90. 
11. Cheon CK, Lim SH, Kim YM, Kim D, Lee NY, Yoon TS, Kim NS, Kim E, Lee JR. Autosomal dominant transmission of complicated hereditary spastic paraplegia due to a dominant negative mutation of KIF1A, SPG30 gene. Sci Rep, 2017. 7(1):12527

12. Pennings M, Schouten MI, van Gaalen J, Meijer RPP, de Bot ST, Kriek M, Saris CGJ, van den Berg LH, van Es MA, Zuidgeest DMH, Elting MW, van de Kamp JM, van Spaendonck-Zwarts KY, Die-Smulders C, Brilstra EH, Verschuuren CC, de Vries BBA, Bruijn J, Sofou K, Duijkers FA, Jaeger B, Schieving JH, van de Warrenburg BP, Kamsteeg EJ. KIF1A variants are a frequent cause of autosomal dominant hereditary spastic paraplegia. Eur J Hum Genet, 2019. Epub ahead of print.

13. DECIPHER: Database of Chromosomal Imbalance and Phenotype in Humans using Ensembl Resources. Firth, H.V. et al (2009). Am.J.Hum.Genet 84, 524-533

14. The National Genomics Research and Healthcare Knowledgebase v5, Genomics England. doi:10.6084/m9.figshare.4530893.v5. 2019.

15. Samanta D, Gokden. M. PEHO syndrome: KIF1A mutation and decreased activity of mitochondrial respiratory chain complex. J Clin Neurosci, 2019. 61:298-301.

16. Landrum MJ, Lee JM, Benson M, Brown GR, Chao C, Chitipiralla S, Gu B, Hart J, Hoffman D, Jang W, Karapetyan K, Katz K, Liu C, Maddipatla Z, Malheiro A, McDaniel K, Ovetsky M, Riley G, Zhou G, Holmes JB, Kattman BL, Maglott DR. ClinVar: improving access to variant interpretations and supporting evidence. Nucleic Acids Res, 2018. 46(D1):D1062-67.

17. Krenn M, Zulehner G, Hotzy C, Rath J, Stogmann E, Wagner M, Haack TB, Strom TM, Zimprich A, Zimprich F. Hereditary spastic paraplegia caused by compound heterozygous mutations outside the motor domain of the KIF1A gene. Eur J Neurol, 2017. 24(5):741-747 
18. Tomaselli PJ, Rossor A, Horga A, Laura M, Blake JC, Houlden H, Reilly MM. A de novo dominant mutation in KIF1A associated with axonal neuropathy, spasticity and autism spectrum disorder. JPNS, 2017. 460-463.

19. Wang J, Zhang Q, Chen Y, Yus S, Wu X, Bao X. Rett and Rett-like syndrome: Expanding the genetic spectrum to KIF1A and GRIN1 gene. Mol Genet Genomic Med, 2019. 11:e968

20. Next Generation Sequencing Service, Sheffield Children's NHS Foundation Trust, UK. https://www.sheffieldchildrens.nhs.uk/sdgs/next-generation-sequencing/. Last updated October 2018; accessed October 2019.

21. Genomics England PanelApp. https://panelapp.genomicsengland.co.uk/panels/258/. Published January 2016; accessed October 2019.

\section{Table Legends}

Table 1: Clinical and genetic characteristics of patients. Abbreviations: ADHD: Attention deficit hyperactivity disorder; ASD: autism spectrum disorder; CC: corpus callosum; CSF: cerebrospinal fluid; GMFCSE: Gross Motor Function Classification equivalent (estimated score); GTCS: generalised tonic-clonic seizure; LD: learning difficulty; LL: lower limb; OA: optic atrophy; VEPs: visual evoked potentials; WM: white matter; y: years

\section{Figure Legends}

Figure 1

Case 1 shows callosal agenesis (black arrows $a, b, c$ ), shallow pontomedullary sulcus (white arrow a), incomplete hippocampal inversion (white arrow $c$ ) and optic atrophy (white arrows d). 
Case 2: MRI at seven months old shows hypo/dysgenesis of corpus callosum (white arrow a) and superior vermian volume loss (black arrow $a$ ). Axial T2 $(b)$ shows cerebral volume loss and posterior predominant white matter signal changes (white arrow). Earlier MRI at 10 days old $(c, d)$ shows preservation of cerebral and cerebellar volume (white arrows).

Case 3 demonstrates progressive vermian atrophy (white arrow a) along with posterior callosal volume loss (black arrow a) and posterior predominant white matter changes (white arrow c). Axial T2 image shows cerebellar volume loss (black arrow d) and bilateral dentate hyper intensity (white arrow $d)$.

Case 4 shows progressive cerebellar volume loss (white arrows $a, b$ ), small posterior callosum (black arrow a), posterior white matter hyper intensity (white arrows $c$ ) and mild dentate hyper intensity (white arrow $d$ ).

Case 5A shows moderate cerebellar volume loss (white arrow a) with a slightly thin posterior callosum (black arrow a). The pons seems to be bulky (white arrow $b$ ) with small symmetrical paramedian bumps (white arrow c). Mild posterior white matter signal changes (white arrow $d$ ) as well as dentate hyperintensity (black arrow d) are also seen. 